\title{
A Comparative Study of the Promotion of Tissue Plasminogen Activator and Pro-Urokinase-induced Plasminogen Activation by Fragments D and E-2 of Fibrin
}

Jian-ning Liu and Victor Gurewich

Vascular Research Laboratory, Institute for the Prevention of Cardiovascular Disease, New England Deaconess Hospital, Harvard Medical School, Boston, Massachusetts 02215

\begin{abstract}
Plasmin generation by equimolar concentrations of tissue plasminogen activator (t-PA), pro-urokinase (pro-UK), and urokinase (UK), and a twofold higher concentration of a plasmin-resistant mutant rpro-UK (Ala-158-pro-UK) was measured on a microtiter plate reader. The promoting effects on this reaction of equimolar concentrations of fibrinogen, soluble fibrin (Desafib), CNBr fragment FCB-2 (an analogue of fragment D), or purified fragment E-2 were compared. Plasmin generation by t-PA was moderately promoted by fibrinogen, substantially promoted by Desafib and FCB-2, but not at all promoted by fragment E-2. By contrast, plasmin generation by pro-UK or by Ala-158-pro-UK was not promoted either by fibrinogen, Desafib, or FCB-2, but was significantly promoted by fragment E-2. Plasmin generation by UK was not significantly promoted by any of the fibrin(ogen) preparations. Treatment of fragment E-2 by carboxypeptidase-B (CPB), eliminated its promotion of pro-UK and Ala-158-pro-UK-induced plasmin generation. Pretreatment of FCB-2 with plasmin slightly potentiated its promotion of $t-P A$ activity. This effect of plasmin pretreatment of FCB-2 was reversed by CPB treatment. Plasmin pretreatment of FCB-2 did not induce any promotion of activity in pro-UK or Ala-158-pro-UK.

The findings show that the intrinsic activity of pro-UK and the activity of $t-P A$ are promoted by different regions of the fibrin(ogen) molecule. The latter is stimulated primarily by a determinant in the fragment $D$ region, which is available in intact fibrin. By contrast, plasminogen activation by the intrinsic activity of pro-UK was stimulated exclusively by fragment E-2, which is unavailable in intact fibrin. The findings are believed relevant to fibrinolysis and support the concept that t-PA and pro-UK are complementary, sequential, and synergistic in their actions. (J. Clin. Invest. 1991. 88:2012-2017.) Key words: plasmin • carboxy-terminal • internal $\bullet$ lysine $\bullet$ residues
\end{abstract}

\section{Introduction}

In previous studies of clot lysis in a plasma milieu, it was shown that tissue plasminogen activator (t-PA) ${ }^{1}$ and pro-urokinase

Address reprint requests to Victor Gurewich, M.D., Vascular Research Laboratory, Burlington Bldg., New England Deaconess Hospital, 185 Pilgrim Road, Boston, MA 02215.

Received for publication 14 February 1991 and in revised form 15 August 1991.

1. Abbreviations used in this paper: $\mathrm{CPB}$, carboxypeptidase-B; pNA, $p$-nitro analine; pro-UK, pro-urokinase; t-PA, tissue plasminogen activator.

J. Clin. Invest.

(c) The American Society for Clinical Investigation, Inc. 0021-9738/91/12/2012/06 \$2.00

Volume 88, December 1991, 2012-2017
(pro-UK) had certain complementary properties at fibrin-specific doses. Clot lysis by t-PA was more linear and relatively unaffected by plasmin pretreatment of the clot. By contrast, clot lysis by pro-UK was preceded by a lag phase which was strongly attenuated by plasmin-pretreatment of fibrin. This effect of plasmin could be reversed by subsequent treatment of the clot with carboxypeptidase-B (1). These findings appeared to suggest that t-PA and pro-UK activated different plasminogen substrates in the fibrin clot. It was proposed that t-PA predominantly activated plasminogen bound to intact fibrin, as previously suggested by Verheijen et al. (2), whereas pro-UK activated plasminogen bound to $\mathrm{COOH}$-terminal lysines, either on the E-domain or on the $\alpha$-chain of partially degraded fibrin.

The present study was undertaken to critically examine this hypothesis in a purified system by comparing the promoting effects of fibrinogen, CNBr fragment FCB-2 (an analogue of fragment $D$ ), and purified fragment E-2 on plasminogen activation by t-PA, pro-UK, a plasmin-resistant mutant rpro-UK (Ala-158-pro-UK), and urokinase (UK).

\section{Methods}

Materials. Pro-UK, purified from a transformed human kidney cell line, was obtained from Collaborative Research Inc. (Lexington, MA). It contained $<1 \%$ two-chain $\mathrm{UK}$ and was further purified by treatment with DFP as previously described (3). A plasmin-resistant mutant form of r-pro-UK was constructed by site-directed mutagenesis of Lys$158 \rightarrow$ Ala-158 (Ala-158-pro-UK) and was obtained from the same source. Two-chain, $55 \mathrm{kD}$ UK was obtained from Green Cross (Osaka, Japan). Single chain t-PA was obtained from Boehringer Mannheim GmbH (Mannheim, Germany). The concentration of the plasminogen activators was standardized with the t-PA and UK International Reference Preparations (National Institute for Biological Standards and Controls, London, UK). For this purpose the pro-UK was first converted to UK by incubation with plasmin as previously described (4). The concentration of the Ala-158-pro-UK was determined from the absorbance using the extinction coefficient of pro-UK $\left(E_{280 \mathrm{~mm}}^{18}=13.6\right)$. The Ala-158-pro-UK had an activity against synthetic substrate or against plasminogen that was $0.5 \%$ that of $\mathrm{UK}$, which is similar to the $0.4 \%$ reported by Pannell et al. (3) and Petersen et al. (5) for the intrinsic activity of pro-UK. Carboxypeptidase-B (CPB) was obtained from Calbiochem-Behring Corp. (La Jolla, CA). Purified human thrombin was a gift from Dr. John Fenton (Albany, NY). Glu-Plasminogen was purified from DFP-treated bank plasma essentially by the method of Castellino and Powell (6). Fibrinogen and synthetic chromogenic substrates for plasmin (S2251) and for UK (S2444) were obtained from Kabi Pharmacia, Inc. (Franklin, $\mathrm{OH}$ ). A CNBr fragment of fibrinogen (FCB-2), which is an analogue of fragment $\mathrm{D}$, soluble fibrin (Desafib), and Lys-plasmin were obtained from American Diagnostica Inc. (Greenwich, CT).

Preparation of fragment E-2. Fragment E-2 was selected from among the fragments E characterized by Olexa et al. (7) because it can be purified most readily and because it is relatively stable.

Fragment E-2 was prepared by a modification of the method of Varadi and Patthy (8) as follows: A plasmin digest of fibrin was prepared by dissolving human fibrinogen in $0.05 \mathrm{M}$ Tris- $\mathrm{HCl}, 0.15 \mathrm{M}$ 
$\mathrm{NaCl}-0.01 \mathrm{M} \mathrm{CaCl}_{2}, \mathrm{pH} 7.4$, at a final concentration of $10 \mathrm{mg} / \mathrm{ml}$. The solution was equilibrated to $37^{\circ} \mathrm{C}$ and Glu-plasminogen $(0.025 \mathrm{mg} / \mathrm{ml})$ and streptokinase (Hoechst Pharmaceuticals, Somerville, NJ) (7.5 IU/ $\mathrm{ml})$ were added immediately before the addition of highly purified thrombin $(8 \mathrm{NIH} \mathrm{U} / \mathrm{ml})$. The reaction mixture was incubated $\left(37^{\circ} \mathrm{C}\right)$ for $3 \mathrm{~h}$. Digestion was arrested by the addition of aprotinin (Trasylol, $100 \mathrm{KIU} / \mathrm{ml}$; Miles Inc., West Haven, CT), epsilonaminocaproic acid $(0.02 \mathrm{M})$, and EDTA $(0.02 \mathrm{M})$.

The digest was then applied onto a Sephacryl S-200 (Pharmacia Fine Chemicals, Piscataway, NJ) column $(2.5 \times 160 \mathrm{~cm})$ equilibrated with $0.3 \mathrm{M} \mathrm{NH}_{4} \mathrm{HCO}_{3}$ and eluted with the same buffer. Fragments $\mathrm{E}$ were obtained from pool B, whereas pool A contained DD-E. The latter pool was lyophilized and dissolved in $0.05 \mathrm{M}$ sodium citrate, $3 \mathrm{M}$ urea, pH 5.5, and incubated $\left(37^{\circ} \mathrm{C}\right)$ for $3 \mathrm{~h}$ as described by Varadi and Patthy (9). Fragment E-2 was separated from DD-E by gel filtration on a Sephacryl S-300 column $(2.5 \times 160 \mathrm{~cm})$ equilibrated with $0.05 \mathrm{M}$ sodium citrate, $3 \mathrm{M}$ urea. The purified E-2 preparation was desalted and lyophilized.

The preparation was tested for plasmin activity with S2251 and trace activity was found. To remove this, the preparation was treated with EACA $(0.5 \mathrm{M})$ and diisopropylflurophosphate $(5 \mathrm{mM})$ for $1 \mathrm{~h}$ $\left(37^{\circ} \mathrm{C}\right)$, and then dialyzed exhaustively against buffer $(0.05 \mathrm{M}$ sodium phosphate, $0.15 \mathrm{M} \mathrm{NaCl}, \mathrm{pH} 7.8$ ).

The concentration of fragment E-2 was determined from the absorbance $(280 \mathrm{~nm})$ divided by the molecular extinction coefficient of fragment $E-2\left(E_{280 \mathrm{~mm}}^{1 \%}=10.0\right)$. It was additionally measured using a protein assay kit (Bio-Rad Laboratories, Inc., Richmond, CA). The final preparation was examined by gradient (5-15\%) SDS-PAGE.

Plasmin and CPB treatment. (a) Plasmin-treatment of FCB-2: 20 $\mu \mathrm{M}$ FCB-2 was dissolved in $0.05 \mathrm{M}$ sodium phosphate, $0.15 \mathrm{M} \mathrm{NaCl}$,

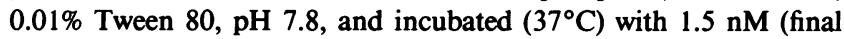
concentration) plasmin for $5 \mathrm{~min}$, after which DFP ( $5 \mathrm{mM}$, final concentration) was added and incubated $\left(37^{\circ} \mathrm{C}\right)$ for $1 \mathrm{~h}$. The mixture was dialyzed with $0.05 \mathrm{M}$ sodium phosphate, $0.15 \mathrm{M} \mathrm{NaCl}, 0.01 \%$ Tween 80, $\mathrm{pH}$ 7.8. (b) CPB treatment: $50 \mu \mathrm{M}$ fragment-E2 was dissolved in $0.05 \mathrm{M}$ sodium phosphate, $0.15 \mathrm{M} \mathrm{NaCl}, 0.01 \%$ Tween $80, \mathrm{pH} 7.8$, and incubated $\left(37^{\circ}\right)$ for $1 \mathrm{~h}$ after the addition of $\mathrm{CPB}(0.5 \mu \mathrm{M}$, final concentration). $10 \mu \mathrm{M}$ Desafib, FCB-2, or plasmin-treated-FCB-2 were dissolved in the same buffer and incubated $\left(37^{\circ} \mathrm{C}\right)$ with $\mathrm{CPB}(0.1 \mu \mathrm{M}$ final concentration) for $1 \mathrm{~h}$ at $37^{\circ} \mathrm{C}$, a concentration and time sufficient to remove most of the $\mathrm{COOH}$-terminal lysines (1). In the experiments with FCB-2 and Desafib, the CPB was included in the reaction mixture to remove any generated $\mathrm{COOH}$-terminal lysines.

Evaluation of the effects of fibrin(ogen) or its derivatives on the plasmin activity assay. The effect of the stimulators (fibrin[ogen] and its derivatives) on the plasmin assay was evaluated. A reaction mixture consisting of $1 \mu \mathrm{M}$ stimulator, $1.5 \mathrm{mM} \mathrm{S} 2251$, and plasmin (0-100 $\mathrm{nM}$ ) in $0.05 \mathrm{M}$ sodium phosphate, $0.15 \mathrm{M} \mathrm{NaCl}, 0.2 \% \mathrm{BSA}, 0.01 \%$ Tween 80, pH 7.8 was incubated at room temperature. The concentration-slope curves in the presence and absence of the stimulators was measured by the $O D$ increase with time at $410 \mathrm{~nm}$ against a reference wavelength of $490 \mathrm{~nm}(410 / 490 \mathrm{~nm})$ on a microtiter plate reader $\left(M_{R}\right.$ 5000; Dynatech Laboratories, Inc., Alexandria, VA).

Effects of fibrin(ogen) or its derivatives on plasmin generation by plasminogen activators. The time-absorbance curves of the stimulation by fibrinogen, FCB-2, Desafib and fragment E-2 on plasminogen activation by t-PA, pro-urokinase, Ala-158-pro-UK, and UK were determined by measuring the $O D$ increase in the reaction mixture with time at $410 \mathrm{~nm}$ against a reference wavelength of $490 \mathrm{~nm}(410 / 490 \mathrm{~nm})$ on the microtiter plate reader. The reaction mixture contained $1.5 \mathrm{mM}$ S2251, $0.24 \mu \mathrm{M}$ Glu-plasminogen, $1 \mu \mathrm{M}$ of the stimulator and $0.5 \mathrm{nM}$ of the plasminogen activator (except for Ala-158-pro-UK which was $1.0 \mathrm{nM}$ ) in $0.05 \mathrm{M}$ sodium phosphate, $0.15 \mathrm{M} \mathrm{NaCl}, 0.2 \% \mathrm{BSA}, 0.01 \%$ Tween 80, pH 7.8 at room temperature. The effect of plasmin and CPB treatment of the stimulators was also evaluated. Each experiment was done in quadruplicate and all comparisons were made only of data from the same experiment. At the concentrations used, neither UK nor t-PA had any effect on S2251.
Methodology for determining the reaction rates of the activators. The rate of each of the reactions was determined from the absorbance as a function of time, expressed as follows:

$A(t)_{\mathrm{i}}=V_{\mathrm{i}} \cdot t^{2}+B$,

where $V_{\mathrm{i}}$ is the reaction rate and $B$ the background $\left(t_{\mathrm{o}}\right.$ absorbance). This equation is based on the following analysis:

Plasminogen activation by t-PA, UK, or Ala-158-pro-UK, coupled with measuring plasmin generation with $\mathbf{S} 2251$, can be described by the following two reactions:

Plasminogen $\stackrel{\text { PA }}{\rightarrow}$ Plasmin

S-2251 $\stackrel{\text { Plasmin }}{\longrightarrow}$ Peptide + pNA $\left(\Delta A_{410 / 490 \mathrm{~nm}}\right)$.

The time-absorbance curves of these reactions can be plotted from the assays. The value for the reaction rate can then be determined directly by curve fitting using the integrated rate equation adapted from Chibber et al. (10).

$A(t)_{\mathrm{i} 410 / 490 \mathrm{~nm}}=\frac{\epsilon D}{2} \times \frac{k_{\mathrm{cat}, \mathrm{PA}}[\mathrm{PA}][\mathrm{Plg}]_{\mathrm{i}}}{K_{\mathrm{m}, \mathrm{PA}}+[\mathrm{Plg}]_{\mathrm{i}}} \cdot t^{2}+B$,

where $\epsilon$ is the molar extinction coefficient for $p$-nitro analine (pNA) in a $100-\mu \mathrm{l}$ well, $A$ is absorbance and subscript i represents the concentration used, $B$ is the value for the background $\left(B=A_{t-0}\right)$, and

$D=\frac{k_{\mathrm{cat}, \mathrm{pl}}[\mathrm{S} 2251]}{K_{\mathrm{m}, \mathrm{pl}}+[\mathrm{S} 2251]}$

$V_{\mathrm{i}}=\frac{\epsilon D}{2} \times \frac{k_{\mathrm{cat}, \mathrm{PA}}[\mathrm{PA}][\mathrm{Plg}]_{\mathrm{i}}}{K_{\mathrm{m}, \mathrm{PA}}+[\mathrm{Plg}]_{\mathrm{i}}}$

Curve fitting was performed on early time points, before depletion of substrates became significant, using the Enzfitter program (ElsevierBiosoft, Cambridge, UK). The $V_{i}$ was directly obtained by nonlinear regression analysis.

Although pro-UK is problematic in that it is converted to UK during the course of the assay, for the purpose of the comparison, the same analytic procedure was used. However, to minimize the effect of UK generation, analysis was restricted to earlier time points ( $<120 \mathrm{~min}$ ).

Effect of fragment E-2 on plasmin generation by Ala-158-pro-UK. Time absorbance curves of plasminogen activation by Ala-158-proUK were obtained in the presence of a range of concentrations (0-20 $\mu \mathrm{M})$ of fragment E-2 using the method described above. The concentration of the other reactants was not changed from that used in the other experiments. The reaction mixture contained S-2251 (1.5 mM), Glu-plasminogen $(0.24 \mu \mathrm{M})$, and Ala-158-pro-UK ( $1.0 \mathrm{nM})$ and was incubated for $60 \mathrm{~min}$. The promotion of the rate of plasmin generation by fragment E-2 $(2.5,5,10,20 \mu \mathrm{M})$ was determined. The results were expressed as a ratio of the reaction rate in the presence versus the absence of the ligand.

In a control experiment, FCB-2 $(20 \mu \mathrm{M})$ was substituted for fragment E-2.

\section{Results}

Characterization of the fragment $E-2$ preparation by SDS$P A G E$ (Fig. 1). The fragment E was purified from the DD-E complex and therefore corresponded to either fragment E-1 or E-2, because fragment E-3 does not bond to DD (7). The preparation was homogenous and fragment E-1 and E-2 were resolved by SDS-PAGE. Moreover, the final preparation was shown to comigrate with a commercial preparation of fragment E-2 (American Bioproducts, Parsippany, NJ) (data not shown). A trace plasmin contaminant in the preparation was removed as described above. 


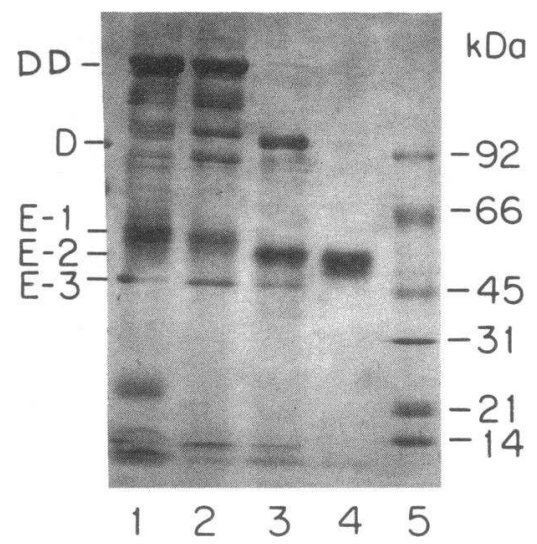

ments D, E-2, and E-3; (lane 4) fractions from column 2 pool B containing purified fragment E-2; (lane 5) molecular weight markers.

Fig. 1 shows the fibrin digest and purification steps on gradient (5-15\%) SDS-PAGE. Lane 1 shows the fibrin digestion mixture containing the DD-E complex which dissociates to DD dimer and $\mathrm{E}$ fragment in SDS, fragments $\mathrm{D}$ and $\mathrm{E}$. Lane 2 shows selected fraction pools from column 1 containing the DD-E complex (which is DD dimer and E fragment in SDSPAGE) and lane 3 shows fragments D, E-2, and E-3. Lane 4 shows the purified fragment E-2 from column 2 . Lane 5 contains molecular weight markers.

Effect of fibrin(ogen) and its derivatives on the detection of plasmin by the assay (Table I). Certain fibrin(ogen) derivatives may compete with the synthetic substrate for plasmin or may change the reactivity of plasmin through a conformational change, therefore, the effect of the ligands on the plasmin assay was evaluated. A range of plasmin concentrations (0-100 nM) were tested. Only fragment E-2 induced a slight stimulation in the plasmin assay relative to the buffer control value, whereas Desafib and FCB-2 induced slight inhibition, which was greatest at lower concentrations of plasmin (Table I).

Promotion of plasmin generation by fibrin(ogen) and its derivatives (Figs. 2, $A-D$ ). Plasmin generation by pro-UK was promoted only by fragment E-2 (curve 1 ). The other fragments induced slight inhibition (curves 3-5) compared with the buffer control (curve 2). This slight inhibition was probably due to the effect of these fragments on the plasmin assay (see above) (Fig. $2 A$ ).

Negligible plasminogen activation was seen with the Ala158-pro-UK at twice the concentration of the other activators, reflecting the low intrinsic activity of this mutant and its resistance to plasmin activation (curve 3). However, in the presence of fragment E-2, significant plasmin generation occurred (curve 1), the promotion being qualitatively comparable to that obtained with pro-UK (Fig. $2 \mathrm{~B}$ ). The negative OD is related to the background noise.

The quantitative difference, reflected by the scales used on the $y$-axis of Fig. 2, $A$ and $B$, is due to the fact that pro-UK but not Ala-158-pro-UK is activated during the time course, thereby amplifying plasmin generation.

By contrast, t-PA induced plasminogen activation was not promoted by fragment E-2 (curve 5), being identical to the buffer control (curve 4). Instead, substantial promotion of plasmin generation was induced by FCB-2 (curve 1 ) and Desa-
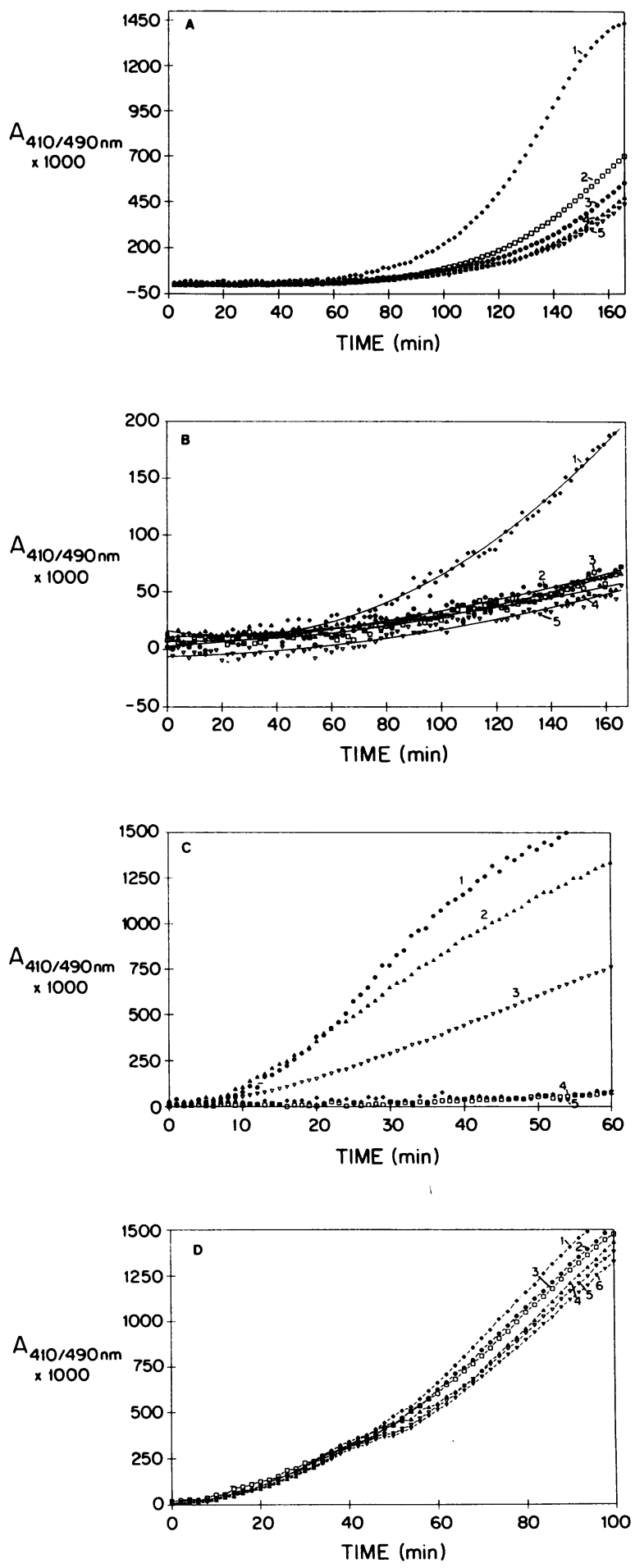

Figure 2. $(A)$ Plasmin generation by pro-UK $(0.5 \mathrm{nM})$ in buffer containing Glu-plasminogen $(0.24 \mu \mathrm{M})(\square)$ and in the presence of fragment E-2 $(\bullet)$, FCB-2 $(\bullet)$, Desafib $(\triangleleft)$, or fibrinogen $(\nabla) .(B)$ Plasmin generation by Ala-158-pro-UK ( $1 \mathrm{nM})$ in buffer containing Glu-plasminogen $(0.24 \mu \mathrm{M})(\square)$ and in the presence of fragment E-2 $(\bullet)$,

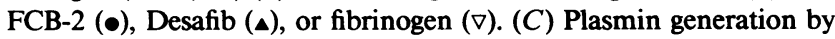
t-PA $(0.5 \mathrm{nM})$ in buffer containing Glu-plasminogen $(0.24 \mu \mathrm{M})(\square)$, and in the presence of FCB-2 (•), Desafib ( $\Delta)$, fibrinogen $(\nabla)$ or fragment E-2 ( $)$. (D) Plasmin generation by UK $(0.5 \mathrm{nM})$ in buffer containing Glu-plasminogen $(0.24 \mu \mathrm{M})(\square)$, and in the presence of fragment E-2 $(\bullet)$, FCB-2 $(\bullet)$, Desafib $(\triangleleft)$, fibrinogen $(\nabla)$, or with fibrinogen in the presence of CPB $(\nabla)$. 
fib (curve 2). A moderate promotion of plasmin generation was also induced by fibrinogen (curve 3) (Fig. $2 C$ ).

Plasmin generation by UK (curve 3 ) was not significantly promoted by FCB-2 (curve 2) or any of the fibrin(ogen) preparations. The slight promotion by fragment E-2 (curve 1) and slight inhibition by Desafib (curve 4), fibrinogen (curve 5), and CPB-treated fibrinogen (curve 6) being similar to the effect induced by the ligands on the assay as shown in Table I (Fig. $2 D$ ).

Effect of plasmin and CPB treatment of FCB-2, Desafib, and fibrinogen on plasmin generation by t-PA (Fig. 3 A). Pretreatment of FCB-2 with plasmin in order to expose a $\mathrm{COOH}$ terminal lysine, slightly but reproducibly, potentiated its promotion of plasmin generation by t-PA (curve 1 vs. 2). This effect was reversed by subsequent treatment of FCB-2 with CPB (curve 3). Similarly, CPB attenuated the promotion of plasmin generation by Desafib (curve 4 vs. 5). CPB treatment of fibrinogen had no effect on its promotion of plasmin generation by t-PA (curve 6 vs. 7). In the absence of ligands, little plasmin generation occurred (curve 8 ) (Fig. $3 \mathrm{~A}$ ).

$C P B$ treatment of fragment $E-2$ and plasmin pretreatment of FCB-2 on plasmin generation by pro-UK and Ala-158-pro$U K$ (Fig. 3, $B$ and C). CPB treatment of fragment E-2 (curve 1), to remove the $\mathrm{COOH}$-terminal lysines, eradicated its promotion of plasmin generation by pro-UK (curve 2) bringing it down to that of the buffer control (curve 3). Plasmin pretreated FCB-2 had no effect on pro-UK activity and plasmin generation remained below (curve 4) that of the buffer control, indicating that the $\mathrm{A} \alpha$-chain $\mathrm{COOH}$-terminal lysine which augmented t-PA activity (see Fig. $3 A$ ) had no effect on pro-UK activity (Fig. $3 \mathrm{~B}$ ).

The results obtained with the Ala-158-pro-UK were similar. The CPB treatment of fragment E-2 (curve 1) eradicated its promotion of plasmin generation (curve 2) which became comparable to the buffer control (curve 3). Plasmin pretreatment of FCB-2 did not induce any promotion of plasmin generation (curve 4). Only quantitative differences from pro-UK were seen due to the nonactivatability of the mutant (Fig. $3 \mathrm{C}$ ).

Control experiments with CPB showed that it had no effect on plasmin generation by any of the activators.

Comparison of the reaction rates of the activators in the presence of fibrin(ogen) and its derivatives (Table II). A comparison of the reaction rates (mean $\pm \mathrm{SD}$ ) of $\mathrm{t}-\mathrm{PA}, \mathrm{UK}$, pro-UK, and Ala-158-pro-UK in the presence or absence of fibrin(ogen)

Table I. Effect of Fibrin(ogen) Derivatives on the Plasmin Assay Expressed as a Fraction of the Buffer Control Value

\begin{tabular}{rrrrr}
\hline Plasmin & Buffer & $\begin{array}{c}\text { E-2 } \\
(1 \mu M)\end{array}$ & $\begin{array}{c}\text { Desafib } \\
(1 \mu M)\end{array}$ & $\begin{array}{c}\text { FCB-2 } \\
(1 \mu M)\end{array}$ \\
\hline$n M$ & & & & \\
3 & 1.00 & 1.101 & 0.645 & 0.699 \\
5 & 1.00 & 1.087 & 0.664 & 0.751 \\
8 & 1.00 & 1.072 & 0.688 & 0.807 \\
10 & 1.00 & 1.056 & 0.716 & 0.850 \\
30 & 1.00 & 1.044 & 0.790 & 0.892 \\
50 & 1.00 & 1.030 & 0.869 & 0.927 \\
80 & 1.00 & 1.023 & 0.939 & 0.961 \\
100 & 1.00 & 1.012 & 0.990 & 0.998 \\
& & & & \\
\hline
\end{tabular}
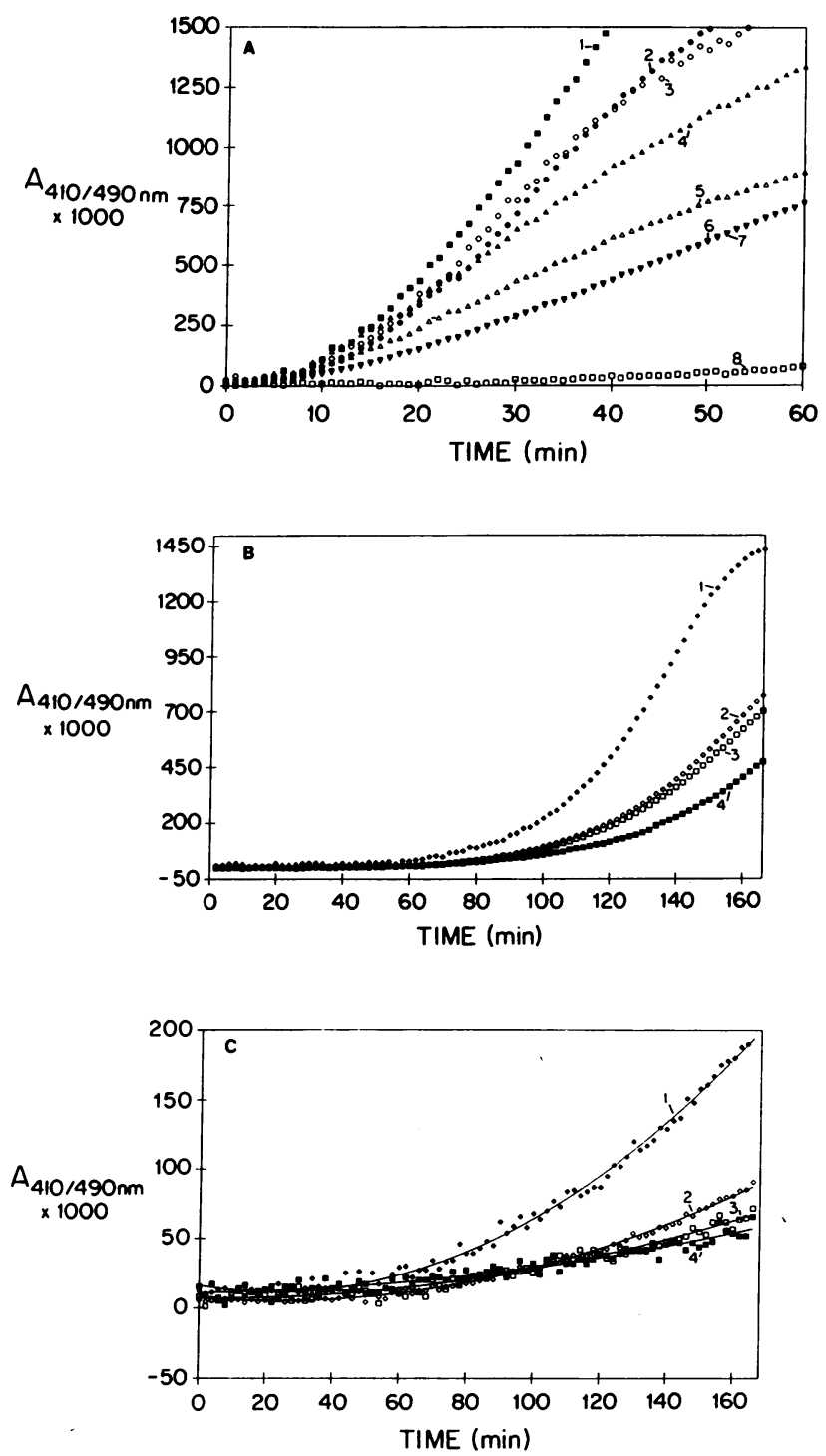

Figure 3. $(A)$ Effect of plasmin-pretreatment and of $\mathrm{CPB}$ on the promotion of t-PA $(0.5 \mathrm{nM})$ activity by fibrin $($ ogen $)$ fragments $(1.0 \mu \mathrm{M})$. Plasmin generation in buffer containing Glu-plasminogen $(0.24 \mu \mathrm{M})$ $(\square)$, and in the presence of plasmin-pretreated FCB-2 ( $\bullet$ ), FCB-2 (•), CPB treatment of plasmin-pretreated FCB-2 (O) Desafib ( $\triangle$ ), CPB plus Desafib $(\Delta)$, or fibrinogen $( \pm C P B)(\nabla \nabla) .(B)$ Effect of plasmin-pretreatment and of CPB on the promotion of pro-UK $(0.5 \mathrm{nM})$ activity by fibrin(ogen) fragments $(1.0 \mu \mathrm{M})$. Plasmin generation in buffer ( $\square$ ) and in the presence of fragment E-2 (४), CPB-treated fragment E-2 $(\diamond)$, plasmin pretreated FCB-2 ( $)$. (C) Effect of plasmin-pretreatment and of CPB on the promotion of Ala-158-pro-UK $(1.0 \mathrm{nM})$ activity by fibrin(ogen) fragments $(1.0 \mu \mathrm{M})$. Plasmin generation in buffer (ם) and in the presence of fragment E-2 (४), CPB-treated fragment E-2 $(\diamond)$, plasmin-pretreated FCB-2 (ם).

and its derivatives, with or without CPB treatment, is shown in Table II. The rate was obtained from the absorbance $\left(\triangle A_{410 / 490 \mathrm{~nm}} \times 10^{6} / \mathrm{min}^{2}\right)$ and curve fitting as described above.

The rate of t-PA-induced plasmin generation was significantly promoted by all fragment D-bearing ligands (FCB-2, Desafib, fibrinogen). There was significant potentiation seen in association with plasmin-pretreatment of FCB-2, an effect which was nullified by CPB. The maximum rate obtained for 
Table II. Comparison of the Reaction Rates $\left(\Delta A_{410 / 490} \times 10^{6} / \mathrm{min}^{2}\right)$ Obtained with the Activators Alone (Buffer) and with the Ligands, with and without Plasmin (pl) or CPB Treatment

\begin{tabular}{|c|c|c|c|c|}
\hline & t-PA & Pro-UK/UK* & Ala $^{158}$-pro-UK & UK \\
\hline Buffer & $13.0 \pm 2.2$ & $23.2 \pm 1.1$ & $2.4 \pm 0.1$ & $591.5 \pm 7.3$ \\
\hline FCB-2 & $781.0 \pm 15.6$ & $17.7 \pm 0.9$ & $2.2 \pm 0.1$ & $625.2 \pm 5.1$ \\
\hline pl-FCB-2 & $1001.3 \pm 8.4$ & $17.0 \pm 0.1$ & $2.2 \pm 0.1$ & ND \\
\hline $\mathrm{CPB}+\mathrm{pl}-\mathrm{FCB} 2$ & $741.5 \pm 6.6$ & ND & ND & ND \\
\hline Fibrinogen & $207.9 \pm 5.0$ & $13.3 \pm 0.7$ & $1.6 \pm 0.1$ & $536.3 \pm 8.3$ \\
\hline $\mathrm{CPB}+$ fibrinogen & $205.5 \pm 8.2$ & ND & ND & $513.9 \pm 6.9$ \\
\hline E-2 & $17.5 \pm 0.8$ & $54.0 \pm 2.2$ & $6.6 \pm 0.1$ & $678.2 \pm 7.0$ \\
\hline $\mathrm{CPB}+\mathrm{E} 2$ & ND & $25.7 \pm 1.2$ & $2.9 \pm 0.1$ & ND \\
\hline Desafib & $518.4 \pm 19.8$ & $15.0 \pm 0.8$ & $2.1 \pm 0.1$ & $551.8 \pm 10.0$ \\
\hline CPB + Desafib & $250.5 \pm 11.6$ & ND & ND & ND \\
\hline
\end{tabular}

* Because UK generation influences the reaction rate induced by pro-UK, it has been designated pro-UK/UK $(n=4)$.

t-PA was 1,001.3 \pm 8.4 (with plasmin-pretreated FCB-2) compared with a buffer control rate for t-PA of $13.0 \pm 2.2$, corresponding to a 77 -fold promotion by the ligand.

By contrast, the reaction rates of Ala-158-pro-UK and proUK/UK were significantly promoted (compared with the buffer control value) exclusively by fragment E-2 (when not CPB-treated). However, the absolute rates obtained were far less, being 6.6 \pm 0.1 and $54.0 \pm 2.2$ for Ala-158-pro-UK and proUK/UK respectively, corresponding to a two to threefold promotion. This large quantitative difference at low, equimolar (1 $\mu \mathrm{M})$ concentrations of the ligands is believed to reflect the 20 200 -fold lower $K_{\mathrm{m}}$ of t-PA in the presence of a ligand (0.003$0.33 \mu \mathrm{M})(11,12)$ compared with that of either mutant pro-UK (13) or pro-UK (14). This explanation was supported by the findings shown in Figure 4. The UK reaction rate was $591.5 \pm 7.3$ and was little effected by the ligands (Table II).

Promotion of Ala-158-pro-UK-induced plasminogen activation by fragment $E-2$ (Fig. 4). The ratio of the reaction rates with and without fragment E-2 $\left(V_{+\mathrm{E}-2} / V_{-\mathrm{E}-2}\right)$ was plotted against the fragment E-2 concentration. An approximately linear promotion was obtained which was 148.9-fold at the highest concentration of fragment E-2 $(20 \mu \mathrm{M})$. The latter figure corresponds to a reaction rate which approaches that of UK, because the latter has an activity $\sim 200$-fold greater than that of pro-UK or Ala-158-pro-UK $(3,5)$. The promotion by fragment E-2 was not brought to plateau and remained linear at the highest concentration of fragment E-2 tested (Fig. 4).

In the presence of equivalently high concentrations of FCB$2(20 \mu \mathrm{M})$, less than fivefold potentiation of Ala-158-pro-UK plasminogen activating activity was observed (data not shown).

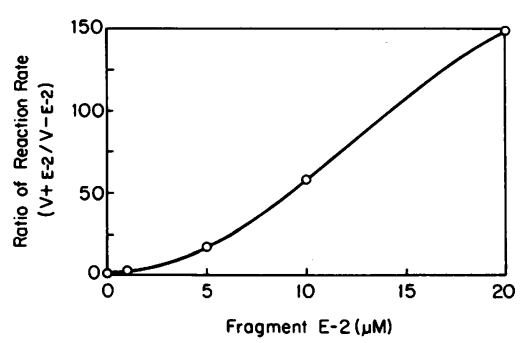

Figure 4. Glu-plasminogen $(0.24 \mu \mathrm{M})$ activation by Ala-158-pro-UK $(1.0 \mathrm{nM})$ in the presence of a range of concentrations of fragment $E-2$ expressed as the promotion of the reaction by the ligand $\left(V_{+\mathrm{E}-2} / V_{-\mathrm{E}-2}\right)$. This ratio is plotted against the ligand concentration.

\section{Discussion}

Plasminogen activation by t-PA is greatly promoted by fibrin to which it has a strong affinity (15). Fibrin(ogen) fragment D (2) containing a critical lysine residue, $\mathrm{A} \alpha-157$ (16), has been specifically implicated in this promotion. This fibrin-mediated stimulation of plasmin generation is critical to the fibrin-specific thrombolytic properties of t-PA. On the other hand, although plasmin generation by pro-UK in plasma has been shown also to be strongly promoted by fibrin (17), the mechanism by which this takes place remains unclear because proUK lacks fibrin affinity.

In studies of clot lysis in a plasma milieu, it was shown that pro-UK, but not t-PA, was relatively inactive against intact fibrin, as reflected by a lag phase. The lag phase was attenuated by plasmin-pretreatment of fibrin, an effect which was reversed by CPB (1). These contrasting fibrinolytic properties of proUK and t-PA suggested that a different fibrin-bound plasminogen was activated by each activator. That is, t-PA appeared to activate principally plasminogen bound to intact fibrin whereas pro-UK activated plasminogen bound to plasmin-exposed $\mathrm{COOH}$-terminal lysines, either on the $\alpha$-chain of fragment D (available on plasmin-treated FCB-2) or on the $\alpha, \beta$, and $\gamma$-chains of fragment $E$.

To evaluate this hypothesis, the effects of equimolar concentrations of several purified fibrin(ogen) preparations on plasminogen activation by $\mathrm{t}-\mathrm{PA}$ or pro-UK were compared. Because pro-UK is itself activated during this reaction by plasmin, a plasmin-resistant pro-UK mutant (Lys-158 $\rightarrow$ Ala-158) was used to isolate the effect of pro-UK itself. The results were compared with those obtained with UK.

Plasminogen activation by t-PA was found to be promoted principally by fibrin(ogen) derivatives containing fragment $D$ (Desafib and FCB-2). This finding is entirely consistent with the previous observations of Verheijen et al. (2) and Voskuilen et al. (16). This promotion was attenuated by the inclusion of $\mathrm{CPB}$ in the reaction mixture, indicating that $\mathrm{COOH}$-terminal lysines generated during the reaction had some augmenting effect. Similarly, pretreatment of FCB-2 by plasmin augmented its potentiation of plasmin generation by t-PA, an effect which was also reversed by CPB. This augmentation by plasmin pretreatment of FCB-2 has been previously reported by Beckman et al. (12) and is believed to be related to the binding of kringle-2 of t-PA by a $\mathrm{COOH}$-terminal lysine as 
suggested by deVries et al. (18). By contrast to fragment $D$, the $\mathrm{COOH}$-terminal lysines on fragment $\mathrm{E}$ appear not to interact similarly with t-PA, because fragment E-2 had no promoting effect on plasminogen activation by t-PA.

In contrast to t-PA, plasminogen activation by pro-UK or Ala-158-pro-UK was not stimulated by FCB-2, Desafib or fibrinogen, indicating that pro-UK did not preferentially activate plasminogen bound to intact fibrin on the $D$ fragment. Moreover, plasmin pretreatment of FCB-2 had no effect, indicating that a $\mathrm{COOH}$-terminal lysine on partially degraded fragment $\mathrm{D}$ is not one of the $\mathrm{COOH}$-terminal lysines involved in the promotion of plasminogen activation by pro-UK. Instead, plasminogen activation by pro-UK or by Ala-158-pro-UK was significantly promoted only by fragment E-2. The stimulation of Ala-158-pro-UK was particularly noteworthy because it had no measurable activity against plasminogen in the absence of fragment E-2. Stimulation by fragment E-2 was lost in the presence of CPB.

Therefore, it appears that the promotion of pro-UK-induced plasmin generation is specifically related to the $\mathrm{COOH}$ terminal lysine(s) on fragment $\mathrm{E}$, represented in the present study by fragment E-2. Varadi and Patthy have previously shown that all three $\mathrm{COOH}$-terminal lysines are needed for the maintenance of the plasminogen binding site on fragment $\mathrm{E}$ (8), and they may be also needed for the induction of a specific conformational change. A conformational alteration in plasminogen has previously been proposed as the principal mechanism by which the fibrin specificity of pro-UK is mediated (4).

At low, equimolar concentrations of the ligands $(1 \mu \mathrm{M})$, the absolute rate of plasmin generation by Ala-158-pro-UK or proUK in the presence of fragment E-2 was only a fraction of that induced by FCB-2 on t-PA (Table II). This quantitative difference may reflect the $K_{\mathrm{m}}$ of t-PA in the presence of this ligand (15) which is 20-200-fold lower than that of Lys-158 mutant pro-UK (13) or pro-UK (14). This interpretation is supported by the major promotion of Ala-158-pro-UK-induced plasminogen activation which was observed when the concentration of fragment E-2 was increased. At a fragment E-2 concentration of $20 \mu \mathrm{M}$, a 148.9-fold promotion was obtained, causing the reaction rate of Ala-158-pro-UK to approach that UK against Glu-plasminogen, which is 200 -fold higher $(3,5)$. Moreover, the promotion remained linear at the highest concentration of fragment E-2 tested (Fig. 4).

The present findings that $\mathrm{t}-\mathrm{PA}$ and pro-UK are promoted by different fibrin fragments is consistent with certain previous observations. These include the complementary and synergistic fibrinolytic effects obtained with combinations of t-PA and pro-UK (1) or t-PA and a Lys-158 plasmin-resistant mutant pro-UK (Val 158) (19), and the lag phase of pro-UK, but not t-PA-induced clot lysis. Because fragment E-bound plasminogen is available only in partially degraded fibrin, the attenuation of the lag phase of pro-UK by plasmin pretreatment of clots (1) is also consistent with the present findings.

In conclusion, it was shown that t-PA and pro-UK-induced plasmin generation is promoted by entirely different fibrin(ogen) fragments, suggesting that each activator is selective and restricted in its activity against fibrin-bound plasminogen. Neither activator activated the alternate fibrin-bound plasminogen any better than it did unbound plasminogen. Therefore, neither t-PA nor pro-UK may be capable of efficiently activating more than a portion of the available fibrin-bound plasminogen in a clot. This may explain their high dose requirements when used alone in therapeutic thrombolysis. The complementary nature of fibrin-dependent plasminogen activation by $t-$ PA and pro-UK provides a rationale for using them together, especially in a sequential combination.

\section{Acknowledgments}

The authors would like to acknowledge the technical assistance of Wendy Kung and the valuable support of Ralph Pannell, Ph.D., and the help in preparation of the manuscript by Joyce J. Lloyd.

This study was supported in part by a grant from Farmitalia Carlo Erba, Italy.

\section{References}

1. Pannell, R., J. Black, and V. Gurewich. 1988 . The complementary modes of action of tissue plasminogen activator (t-PA) and pro-urokinase (pro-UK) by which their synergistic effect on clot lysis may be explained. J. Clin. Invest. 81:853-859.

2. Verheijen, J. H., W. Nieuwenhuizen, and G. Wigngaards. 1982. Activation of plasminogen by tissue plasminogen activator is increased specifically in the presence of certain soluble fibrin(ogen) derivatives. Thromb. Res. 27:377-385.

3. Pannell, R., and V. Gurewich. 1987. The activation of plasminogen by single-chain urokinase or by two-chain urokinase: a demonstration that single chain urokinase has a low catalytic activity (pro-urokinase). Blood. 69:22-26.

4. Pannell, R., and V. Gurewich. 1986. Pro-urokinase-a study of its stability in plasma and a mechanism for its selective fibrinolytic effect. Blood. 67:12151223.

5. Petersen, L. C., L. R. Lund, K. Dano, L. S. Nielsen, and L. Skriver. 1988. One-chain urokinase-type plasminogen activator from human sarcoma cells is a proenzyme with little or no intrinsic activity. J. Biol. Chem. 263:11189-11195.

6. Castellino, F. J., and J. R. Powell. 1981. Human plasminogen. Methods Enzymol. 80:365-378.

7. Olexia, S. A., A. Z. Budzynski, R. F. Doolittle, B. A. Cottrell, and T. C. Greene. 1981. Structure of fragment E species from human cross-linked fibrin Biochemistry. 20:6139-6145.

8. Varadi, A., and L. Patthy. 1984. $\beta\left(\right.$ Leu $\left._{121}-\mathrm{Lys}_{122}\right)$ segment of fibrinogen is in a region essential for plasminogen binding by fragment E. Biochemistry. 23:2108-2112.

9. Varadi, A., and L. Patthy. 1983. Location of plasminogen-binding sites in human fibrin(ogen). Biochemistry. 22:2440-2446.

10. Chibber, B. A. K., J. P. Morris, and F. J. Castellino. 1985. Effects of human fibrinogen and its cleavage products on activation of human plasminogen by streptokinase. Biochemistry. 24:3429-3434.

11. Nieuwenhuizen, W., M. Voskuilen, A. Vermond, B. Hoegee-de Nobel, and D. W. Traas. 1988. The influence of fibrin(ogen) fragments on the kinetic parameters of the tissue-type plasminogen-activator-mediated activation of different forms of plasminogen. Eur. J. Biochem. 174:163-169.

12. Beckman, R., M. Geiger, and B. Binder. 1988. Plasminogen activation by tissue plasminogen activator in the presence of stimulating $\mathrm{CNBr}$ fragment $\mathrm{FCB}$ 2 of fibrinogen is a two-phase reaction. $J$. Biol. Chem. 263:7176-7180.

13. Lijnen, H. R., B. Van Hoef, L. Nelles, and D. Collen. 1990. Plasminogen activation with single-chain urokinase-type plasminogen activator (scu-PA)-studies with active site mutagenized plasminogen (Ser-740 $\rightarrow$ Ala) and plasmin-resistant scu-PA (Lys-1 58 $\rightarrow$ Glu). J. Biol. Chem. 265:5232-5236.

14. Stump, D. C., H. R. Lijnen, and D. Collen. 1986. Purification and characterization of single-chain urokinase-type plasminogen activator form human cell cultures. J. Biol. Chem. 261:1274-1278.

15. Hoylaerts, M., D. C. Rijken, H. R. Lijnen, and D. Collen. 1982. Kinetics of the activation of plasminogen by human tissue plasminogen activator. J. Biol. Chem. 257:2912-2919.

16. Voskuilen, M., A. Vermond, G. H. Veeneman, J. H. VanBoom, E. A Klasen, N. D. Zegers, and W. Niewenhuizen. 1987. Fibrinogen lysine residue A $\alpha-157$ plays a crucial role in the fibrin-induced acceleration of plasminogen activation catalyzed by tissue plasminogen activator. J. Biol. Chem. 262:5944 5946.

17. Gurewich, V., R. Pannell, S. Louie, R. L. Suddith, and R. Greenlee. 1984 Effective and fibrin-specific clot lysis by a zymogen precursor form of urokinase (pro-urokinase). A study in vitro and in two animal species. J. Clin. Invest. 73:1731-1739.

18. deVries, C., H. Veerman, and H. Pannekock. 1989. Identification of the domains of tissue plasminogen activator involved in the augmented binding to fibrin after limited digestion with plasmin. J. Biol. Chem. 264:12604-12610.

19. Gurewich, V., R. Pannell, R. J. Broeze, and J. I. Mao. 1988. Characterization of the intrinsic fibrinolytic properties of pro-urokinase through a study of plasmin resistant mutant forms produced by site specific mutagenesis of lysine 158. J. Clin. Invest. 82:1956-1962. 